\title{
Deux disques relatifs à la musique bouddhique de Sri Lanka
}

\section{Mireille Helffer}

\section{(2) OpenEdition}

\section{Journals}

Édition électronique

URL : http://journals.openedition.org/ethnomusicologie/1479

ISSN : 2235-7688

\section{Éditeur}

ADEM - Ateliers d'ethnomusicologie

Édition imprimée

Date de publication : 31 décembre 1994

Pagination : 299-301

ISBN : 2-8257-0503-9

ISSN : 1662-372X

\section{Référence électronique}

Mireille Helffer, " Deux disques relatifs à la musique bouddhique de Sri Lanka », Cahiers

d'ethnomusicologie [En ligne], 7| 1994, mis en ligne le 03 janvier 2012, consulté le 25 avril 2019. URL: http://journals.openedition.org/ethnomusicologie/1479

Ce document a été généré automatiquement le 25 avril 2019.

Tous droits réservés 


\title{
Deux disques relatifs à la musique bouddhique de Sri Lanka
}

\author{
Mireille Helffer
}

\section{RÉFÉRENCE}

SRI LANKA, Buddhist Chant I: Maha Pirit - The great chant. Performed by monks of the Madangalla Privena. Recorded by Wolfgang Laade, live recording 1979. One CD 1990, Jecklin Disco JD 651-2, Music of Man Archive ; English commentary, 12 pp. SRI LANKA, Buddhist Chant II : Various rituals. Recorded by Wolfgang Laade, live recording 1976/1977. One CD 1993, Jecklin Disco JD 656-2, Music of Man Archive ; English commentary, $12 \mathrm{pp}$.

1 Alors que les musiques du bouddhisme tibétain bénéficient d'une discographie importante, alors que celles du bouddhisme japonais ou coréen sont, elles aussi, relativement bien connues du monde occidental, par le biais d'enregistrements et de publications, les musiques liées à la pratique du bouddhisme dans le sud-est asiatique ont été jusqu'ici négligées, exception faite du disque Sri Lanka - Musiques rituelles et religieuses OCORA 558552 (réédition CD, C 580037), publié à partir des enregistrements faits en 1979 par Hermann C. Vuylsteke. C'est donc avec intérêt et reconnaissance qu'il convient d'accueillir les deux disques publiés dans la collection Music of Man Archive, à partir des enregistrements réalisés sur le terrain par Wolfgang Laade, grâce à une subvention du Fond national suisse de la recherche scientifique, avec la collaboration du Département des Affaires Culturelles à Sri Lanka.

2 Le premier disque, d'une durée de 73'50, est consacré à un rituel, exécuté dans une maison privée par une dizaine de moines, en mémoire d'un jeune homme décédé un an auparavant. Le matériel publié concerne le début de la cérémonie - un maha pirit - qui dura une nuit complète et il comporte deux passages dont il y a lieu de préciser le contenu et le minutage puisqu'une erreur s'est glissée sur la pochette d'accompagnement 
3 Le premier passage [1], long de 11'44, comporte quatre parties enchaînées exécutées par le célébrant principal :

- 0-1'23: « les trois refuges » - tun sarana - correspond à la prise de refuge dans les « Trois Joyaux » que constituent le Bouddha, la loi bouddhique, la communauté monastique ; la formule en est répétée trois fois.

- 1'25-2'55 : « les cinq préceptes»-pansil -, c'est à dire le rappel de l'engagement par lequel le fidèle bouddhiste promet de s'abstenir de détruire la vie, de prendre ce qui ne lui est pas donné,de toute conduite sexuelle désordonnée, de tout mensonge, de toute consommation de boisson alcoolisée.

- 2'56-5'22 introduit le chant d'un gâthâ qui expose le pouvoir du rituel exécuté et les bénédictions résultant de son accomplissement ; après avoir été énoncé par le soliste, chaque vers du texte est répété par le chœur.

- 5'23-11'44 - anu sâsana - correspond à un prêche parlé du célébrant principal.

4 Après un court prélude instrumental confié à des musiciens professionnels (hevisi), le second passage [2], long de 61'57, fait retour à l'aspect vocal et illustre différents modes de cantillation adoptés par les moines du Madangalla Privena.

- 0-6' débute par l'habituelle batterie de tambours - magul bera - qui précède tout rituel ; elle est suivie par le hände duraya exécuté par le hautbois horanäva, un grand tambour à deux peaux, et une paire de petites cymbales.

- 6'15-9'30 : devata âradhana, prière invitant les divinités du panthéon hindou à partager les mérites du rituel.

- 9'37-12'54 : le chœur conclut la partie précédente en répétant plusieurs fois la formule de prise de refuge déjà énoncée dans le premier passage du disque.

- 12'57-58'36 : Suit l'exposé en langue pali de trois sutta (sanskrit sutra), c'est-à-dire de textes dont le contenu est supposé correspondre aux paroles mêmes du Bouddha pendant son existence terrestre.

Du point de vue musical se trouve illustrée ici une technique de chant responsorial par laquelle les deux solistes et le chœur se doivent de maintenir le son sans aucune interruption au moyen de tuilages systématiques. On remarquera en outre comment la hauteur de la formule mélodique adoptée s'élève progressivement depuis le premier énoncé pour débuter finalement un octave plus haut qu'au départ; il s'agit là d'une illustration marquante du phénomène observé dans de nombreuses traditions religieuses et parfois désigné par le terme "montées systématiques ».

6 Le commentaire accompagnant ce premier enregistrement est clair; il utilise à bon escient les explications fournies par Lynn A. de Silva dans un ouvrage publié en 1974 à Colombo sous le titre Buddhism. Beliefs and Practices in Sri Lanka et on appréciera d'y trouver le texte et la traduction des textes du Tun Sarana et du Pansil, ainsi que les titres des textes énoncés (ce qui permettra aux spécialistes de les retrouver facilement) ; mais W. Laade aurait été bien inspiré de consulter également l'article de Cyril de Silva Kulatillake, paru en 1982 dans le Jahrbuch für musikalische Volks- und Völkerkunde (vol.10 : 20-32) dans lequel on trouve des explications pertinentes sur les rapports entre métrique des textes pâli et ligne mélodique du chant. Il aurait été souhaitable de disposer de quelques illustrations permettant de mieux situer les rares interventions instrumentales et de pouvoir localiser plus précisément le lieu de l'enregistrement.

7 Le deuxième disque, paru trois ans après le premier, dure 77'02" et se présente comme un complément de celui-ci, puisqu'il vise, au moyen de douze extraits exécutés par des 
moines âgés appartenant à des monastères différents, à illustrer d'autres styles de cantillation.

8 Les pièces [1] à [6] ont en effet été enregistrées hors situation par le (ou sous la direction du) vénérable Dhammada Kuluhamana du Malwatta Vihâra à Kandy et manifestent un style plus mélismatique; on y remarquera en particulier [4] et [5] le style dit gana sajjhanaya qui, selon le collecteur, n'est plus pratiqué que dans ce monastère (cf. C. de Silva Kulatillake, ibid. : 25-26).

9 Les pièces [7] à [10], quant à elles, illustrent une tradition différente dont est dépositaire le vénérable Rambukvalle Sumangala Thero, appartenant au même monastère; on y observe [8] le style dit âsana dekê bana dans lequel, après l'exposé du texte de base en pâli, le commentaire en srilankais est fourni par un autre officiant .

10 Enfin, les deux derniers extraits, d'une durée respective de $13^{\prime} 22$ et $26^{\prime} 47$, enregistrés auprès du vénérable Pundit Mohottawa Dhammapala du monastère du Râja Mahâ Vihâra de Mahane à Warakapola fournissent un complément appréciable avec l'illustration d'un mode de récitation aujourd'hui tombé en désuétude.

11 La notice de ce second disque est, elle aussi, tout à fait explicite sur les conditions de récitation des différents textes; elle comprend en outre un utile glossaire, mais, tout comme la première, elle ne fournit aucune information sur les caractéristiques proprement musicales des psalmodies sri lankaises.

12 En résumé, ces deux disques constituent un témoignage précieux des traditions musicales dépouillées conservées à Sri Lanka; sans doute trop austères pour toucher le grand public, ils devraient retenir l'attention de tous ceux qui s'intéressent à Sri Lanka, au bouddhisme, ainsi qu'aux différentes formes de psalmodie religieuse dans le monde. 